\title{
10-Gbit/s Sky-Blue Distributed Feedback Laser Diode-Based Visible Light Communication
}

\author{
Meiwei Kong ${ }^{\dagger}$, Jorge A. Holguin-Lerma ${ }^{\dagger}$ Omar Alkhazragi, Xiaobin Sun, Tien Khee Ng, and Boon S. Ooi ${ }^{*}$ \\ Photonics Laboratory, King Abdullah University of Science and Technology (KAUST), Thuwal 23955-6900, Saudi Arabia \\ ${ }^{\dagger}$ These authors contributed equally \\ *boon.ooi@kaust.edu.sa
}

\begin{abstract}
A novel sky-blue ( $480 \mathrm{~nm}) \mathrm{InGaN}$-based distributed feedback laser diode is developed for high-speed visible light communication. With a 3-dB system bandwidth of $\sim 1.5 \mathrm{GHz}, 10 \mathrm{Gbit} / \mathrm{s}$ is achieved by using orthogonal frequency-division multiplexing technology. $\odot 2020$ The Author(s)
\end{abstract}

\section{Introduction}

The fifth generation $(5 \mathrm{G})$ networks are envisioned as the next big milestone in data communications. The major requirements of $5 \mathrm{G}$ include high-speed data rates of up to $10 \mathrm{Gbit} / \mathrm{s}$, high energy efficiency, and full coverage [1]. In these regards, visible light communication (VLC) has attracted significant attention given its properties of being capable of delivering Gbit/s data rates using the unlicensed broad frequency spectrum of light, while being energyefficient and free from electromagnetic interference $[2,3]$.

A succeeding stage of VLC requires the advancement of existing technology, from the system level to the device level. The innovation of the electro-optical devices, both transmitters and receivers, has the potential to deliver new or improved capabilities for a next generation of VLC, even beyond 5G. Similar to the fiber telecommunication infrastructure, one crucial element is the control and finesse of the wavelength. This control has empowered schemes such as dense wavelength-division multiplexing (DWDM), maximizing the capacity of the communication links [4]. In this scenario, the key player has been the distributed feedback (DFB) laser diode (LD) [5].

While DFB-LDs are now widely available at the telecom wavelengths, they are relatively unexplored at the visible wavelengths. Device development has shown InGaN-based DFB-LDs emitting at violet, blue, and green wavelengths [6-10], but aside from a recent report [11], their application to optical wireless communications is little-known.

In this paper, we demonstrate a novel sky-blue InGaN-based DFB-LD emitting at $\sim 480 \mathrm{~nm}$ with a resolutionlimited narrow linewidth of $\sim 34 \mathrm{pm}$. The device shows a side-mode suppression ratio beyond $40 \mathrm{~dB}$ and optical power above $10 \mathrm{~mW}$ under continuous wave (CW) injection current. We further demonstrate the performance of the DFBLD in high-speed VLC. We employ 16-quadrature amplitude modulation (QAM) orthogonal frequency-division multiplexing (OFDM) technology known for its high spectral efficiency [12]. Power-loading (PL) distribution over selected frequencies allows a demonstration of 10-Gbit/s OFDM signals with a bit error rate (BER) of $2.3 \times 10^{-3}$.

\section{Experimental Setup}

Figure 1(a) illustrates the schematic diagram of the DFB-LD-based VLC system. Real-valued 16-QAM OFDM signals were first generated offline by using a personal computer (PC) and then loaded into an arbitrary waveform generator (AWG, Tektronix AWG70002A). The sampling rate of the AWG was set at 25 GSamples/s and the output amplitude of the 16-QAM OFDM signals was within a peak-to-peak voltage of $500 \mathrm{mV}$. Table 1 lists the main parameters of the OFDM signals. After adjusting the amplitudes of the signals by using an amplifier (AMP, ZHL-42W+) and a variable electrical attenuator (ATT, KT2.5-60/1S-2S), the 16-QAM OFDM signals were superimposed into the $\sim 480 \mathrm{~nm}$ DFBLD via a bias-tee (Bias-T, Tektronix PSPL5580) and a CW bias source (Keithley 2520). The frequency bandwidth range of the AMP, ATT, and Bias-T are $10 \mathrm{MHz}-4.2 \mathrm{GHz}, 0-2.5 \mathrm{GHz}$, and $10 \mathrm{kHz}-15 \mathrm{GHz}$, respectively. The gain of the AMP is $30-40 \mathrm{~dB}$ (typ. $34 \mathrm{~dB}$ ).

The high-order DFB grating fabricated on the surface of an InGaN-based LD is shown in Fig. 1(b). The image of the back facet shows the $2-\mu \mathrm{m}$ ridge waveguide. The DFB-LD cavity length is $605 \mu \mathrm{m}$. The design and fabrication of the device follow the Bragg condition and the steps described elsewhere [9]. As shown in Fig. 1(c), the DFB-LD mounted in a TO-56 brass holder is integrated into a thermoelectric cooler (TEC, SaNoor-SN-LDM-T) to keep the case temperature stable at $16{ }^{\circ} \mathrm{C}$ and avoid overheating. The $\mathrm{CW}$ bias current of the DFB-LD was set at $150 \mathrm{~mA}$ in the experiment. After being collimated by a lens mounted in front of the DFB-LD and transmitted through a $\sim 38-\mathrm{cm}$ air channel, the DFB-LD beam with optical 16-QAM OFDM signals was detected by a high-speed photodiode (PD, Alphalas UPD-50-UP). At the receiver side, an AMP was used to increase the amplitude of the received signal, which generally improves the signal-to-noise ratio (SNR). The received 16-QAM OFDM signals were captured by a mixed 
signal oscilloscope (MSO, Tektronix DPO 72004C) and demodulated offline. The sampling rate of the MSO was set at $100 \mathrm{GSamples} / \mathrm{s}$.

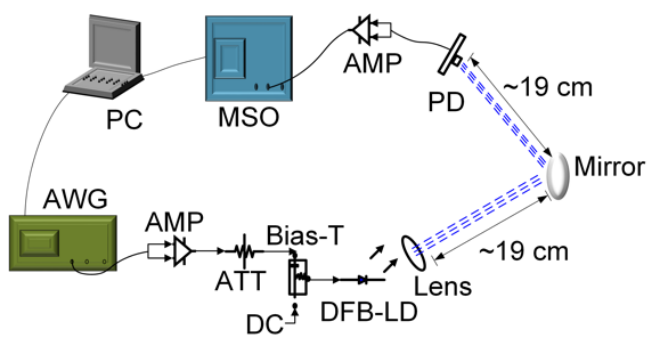

(a)

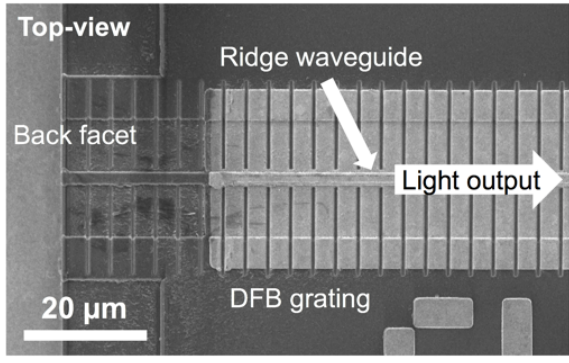

(b)

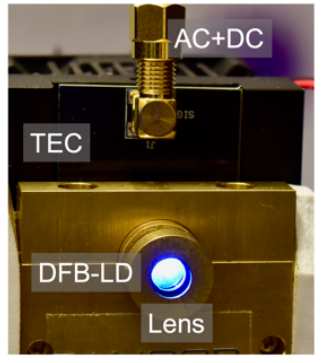

(c)

Fig. 1. (a) Schematic diagram of the DFB LD-based VLC system. (b) Scanning electron microscope top-view image of the high-order DFB grating fabricated on the surface of an InGaN-based LD. (c) DFB-LD mounted on a TEC with the AC+DC signal input at the top.

Table 1. Main parameters of the OFDM signals

\begin{tabular}{cc}
\hline Parameter & Value \\
\hline Data rate of the 16-QAM OFDM signals & 10 Gbit/s \\
Number of subcarriers & 103 \\
Bit number of pseudorandom binary sequence & $2^{20}-1$ \\
Inverse fast Fourier transform size & 1024 \\
Number of cyclic prefix & 10 \\
Number of subcarriers for frequency gap near DC & 3 \\
Number of OFDM symbols (including two training symbols for \\
synchronization and four training symbols for channel equalization)
\end{tabular}

\section{Experimental Results}

The light-current-voltage ( $L-I-V)$ characteristics of the DFB-LD and its optical spectrum at $150 \mathrm{~mA} C W$ bias current are shown in Figs. 2(a) and 2(b), respectively. The peak emission wavelength is located at $\sim 479.7 \mathrm{~nm}$, with a tuning coefficient of $\sim 0.006 \mathrm{~nm} / \mathrm{mA}$. This wavelength falls into the highest transparency window of fresh and seawater (450$550 \mathrm{~nm}$ ), opening the pathway for future applications in underwater wireless optical communication.

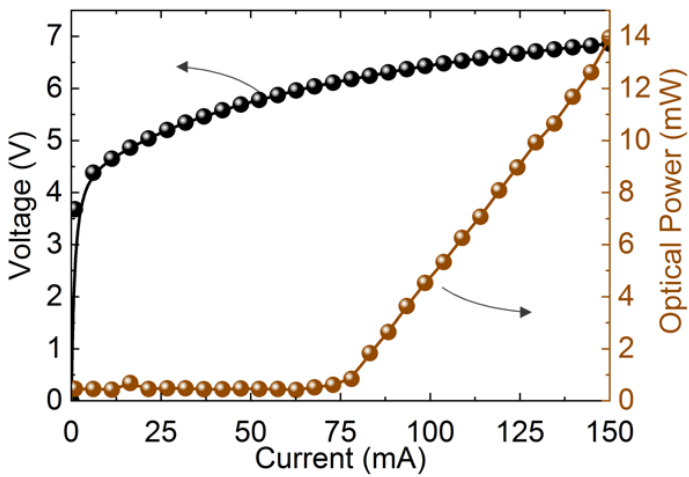

(a)

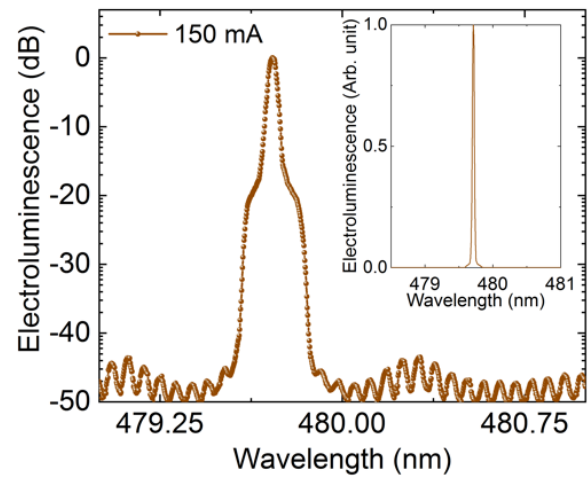

(b)

Fig. 2. (a) CW $L-I-V$ characteristics of the sky-blue DFB-LD and (b) optical spectrum of the sky-blue DFB-LD at $150 \mathrm{~mA} C W$ injection, shown in logarithm scale (the inset figure is the same emission in linear scale).

Figure 3(a) shows the frequency response of the DFB-LD-based system at a transmission distance of $\sim 38 \mathrm{~cm}$, which was measured with a network analyzer (NA, Agilent E8361C PNA). The 3-dB and 10-dB bandwidth of the system are characterized as $\sim 1.5 \mathrm{GHz}$ and $\sim 2.5 \mathrm{GHz}$, respectively. In order to improve these characteristics, which are directly related to the achievable data rate, we enhanced the SNR in the high frequency region by using PL algorithm. 
For subcarriers from the $30^{\text {th }}$ to the $103^{\text {th }}$, the allocated power was linearly emphasized by $4: 11 \mathrm{~dB}$. Under these circumstances, the achieved data rate of the 16-QAM OFDM signals was $10 \mathrm{Gbit} / \mathrm{s}$. The BER for different subcarriers with PL is shown in Fig. 3(b). The BERs in the high-frequency region was improved by the use of PL. The higher BERs at subcarriers of $66^{\text {th }}$ and $67^{\text {th }}$ caused by the non-flat frequency response at the frequency band from $1.66 \mathrm{GHz}$ to $1.73 \mathrm{GHz}$ could potentially be improved in the future by using bit-loading algorithm. Thus, the mean BER was 2.3 $\times 10^{-3}$, which was below the forward error correction (FEC) limit of $3.8 \times 10^{-3}$. Figure 3(c) shows the corresponding well-converged constellation map.

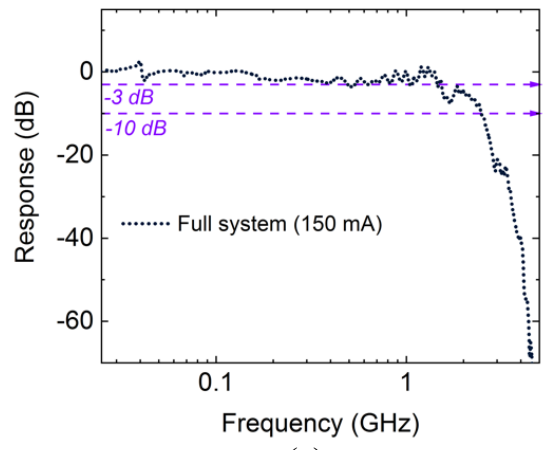

(a)

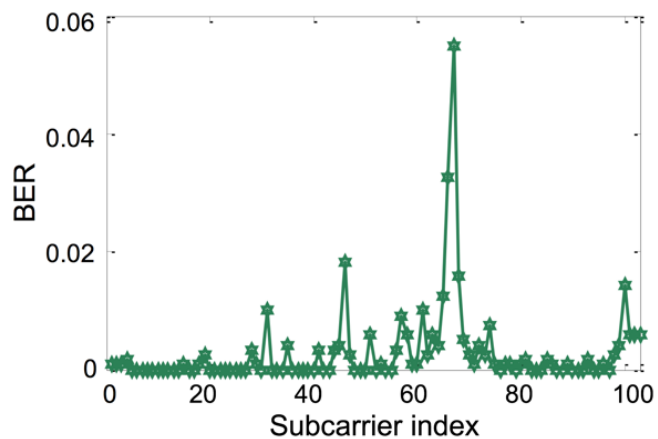

(b)

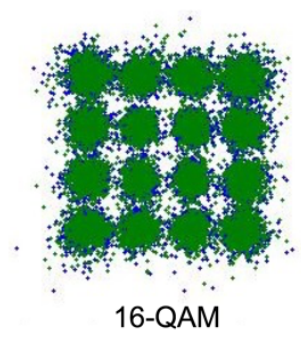

(c)

Fig. 3 (a) Frequency response of the DFB LD-based system at a transmission distance of $\sim 38 \mathrm{~cm}$, (b) BERs for different subcarriers with PL, and (c) the constellation map of the received 16-QAM OFDM signals with a mean BER of $2.3 \times 10^{-3}$.

\section{Conclusions}

In this paper, we experimentally demonstrated the feasibility of using a novel sky-blue DFB-LD to implement highspeed VLC. A data rate of $10 \mathrm{Gbit} / \mathrm{s}$ was achieved by employing OFDM with power-loading algorithm over a distance of $\sim 38 \mathrm{~cm}$ for proof-of-concept. While a single DFB-LD is used in the experiment, the potential utilization of DWDM schemes could lead to record data rates towards the next-generation VLC.

Acknowledgement: KAUST funding OSR-CRG2017-3417, BAS/1/1614-01-01, REP/1/2878-01-01, GEN/1/6607-01-01 and KCR/1/2081-01-01. TKN and BSO gratefully acknowledge funding from KACST, Grant no. R2-FP-008.

\section{References}

[1] M. Agiwal, A. Roy, and N. Saxena, "Next generation 5G wireless networks: a comprehensive survey," IEEE Commun. Surv. Tutorials 18(3), 1617-1655 (2016).

[2] S. Wu, H. Wang, and C.-H. Youn, "Visible light communications for 5G wireless networking systems: from fixed to mobile communications," IEEE Netw. 28(6), 41-45 (2014).

[3] H. Haas, "LiFi is a paradigm-shifting 5G technology," Rev. Phys. 3, $26-31$ (2018).

[4] B. Zhu, T. F. Taunay, M. Fishteyn, X. Liu, S. Chandrasekhar, M. F. Yan, J. M. Fini, E. M. Monberg, and F. V. Dimarcello, "112-Tb/s Space-division multiplexed DWDM transmission with 14-b/s/Hz aggregate spectral efficiency over a 768-km seven-core fiber," Opt. Express 19(17), 16665 (2011).

[5] J. Carroll, J. Whiteaway, and D. Plumb, Distributed Feedback Semiconductor Lasers (IEE-SPIE, 1998).

[6] J. H. Kang, H. Wenzel, V. Hoffmann, E. Freier, L. Sulmoni, R. S. Unger, S. Einfeldt, T. Wernicke, and M. Kneissl, "DFB laser diodes based on GaN using 10th order laterally coupled surface gratings," IEEE Photonics Technol. Lett. 30(3), 231-234 (2018).

[7] T. J. Slight, S. Stanczyk, S. Watson, A. Yadav, S. Grzanka, E. Rafailov, P. Perlin, S. P. Najda, M. Leszczy, S. Gwyn, and A. E. Kelly, "Continuous-wave operation of (Al,In)GaN distributed-feedback laser diodes with high-order notched gratings," Appl. Phys. Express 11, $112701(2018)$

[8] H. Zhang, D. A. Cohen, P. Chan, M. S. Wong, S. Mehari, D. L. Becerra, S. Nakamura, and S. P. DenBaars, "Continuous-wave operation of a semipolar InGaN distributed-feedback blue laser diode with a first-order indium tin oxide surface grating," Opt. Lett. 44(12), 3106 (2019).

[9] J. A. Holguín-Lerma, T. K. Ng, and B. S. Ooi, "Narrow-line InGaN/GaN green laser diode with high-order distributed-feedback surface grating," Appl. Phys. Express 12(4), 042007 (2019).

[10] Deng, Li, Liao, Xie, and Luo, "InGaN/GaN distributed feedback laser diodes with surface gratings and sidewall gratings," Micromachines 10(10), 699 (2019).

[11] S. Gwyn, S. Watson, S. Viola, G. Giuliano, T. J. Slight, S. Stanczyk, S. Grzanka, A. Yadav, K. E. Docherty, E. Rafailov, P. Perlin, S. P. Najda, M. Leszczynski, and A. E. Kelly, "GaN-based distributed feedback laser diodes for optical communications," in Fourth International Conference on Applications of Optics and Photonics, M. F. P. Martins Costa, ed. (SPIE, 2019), (October), p. 1120700.

[12] M. Kong, W. Lv, T. Ali, R. Sarwar, C. Yu, Y. Qiu, F. Qu, Z. Xu, J. Han, and J. Xu, "10-m 9.51-Gb/s RGB laser diodes-based WDM underwater wireless optical communication," Opt. Express 25(17), 20829 (2017). 